\title{
Free dendritic tip growth velocities measured in Al-Ge
}

\author{
M. Becker, ${ }^{1, *}$ S. Klein,${ }^{1, \dagger}$ and F. Kargl ${ }^{1,2, \ddagger}$ \\ ${ }^{1}$ Institut für Materialphysik im Weltraum, Deutsches Zentrum für Luft- und Raumfahrt, 51170 Köln, Germany \\ ${ }^{2}$ Foundry-Institute, RWTH Aachen University, Intzestrasse 5, 52072 Aachen, Germany
}

(Received 11 April 2018; published 30 July 2018)

\begin{abstract}
We determine the tip growth velocities of equiaxed dendrites in an Al-24 at. \% Ge alloy using in situ x-ray radiography. The tip growth velocity is a main characteristic of dendritic growth and allows for an accurate comparison against theories and simulations describing the growth of a single dendrite tip or an array of dendrites. The disk-shaped samples are 200 or $350 \mu \mathrm{m}$ thin and are positioned horizontally in a near-isothermal furnace to suppress buoyancy and gravity-driven melt flows. We obtain a large data set of free dendritic tip growth velocities of Al dendrites over one order of magnitude (4-140 $\mu \mathrm{m} \mathrm{s}^{-1}$ ) and over a decade of global undercooling (1-10 K). No indications for a significant deviation from three-dimensional growth kinetics due to the sample confinement are found.
\end{abstract}

DOI: 10.1103/PhysRevMaterials.2.073405

Equiaxed dendrites are a frequently observed microstructure in metal alloy castings [1,2]. As the microstructure has a strong influence on the performance of a solid, materials scientists and metallurgical engineers are interested to control the final microstructure of a casting to achieve the desired mechanical properties. This requires a deep understanding of the physical processes governing pattern formation, in particular, the evolution of dendritic crystals in metallic alloys. The fundamental concepts of dendritic growth are based on the description of a dendrite tip propagating freely in an undercooled melt, whereby the movement of the solid-liquid interface is controlled by latent heat and solute diffusion away from the interface $[3,4]$. As the aim is to bridge the scales between micro- and macromodeling [5-8], an accurate prediction of dendritic tip kinetics is mandatory, and therefore many theoretical and numerical efforts have been devoted to investigate the steady-state growth of the tip region $[3,9,10]$.

To verify models predicting dendritic growth, experimental in situ observations are necessary. For a proper comparison of equiaxed growth with theoretical models, the measurement of free dendrite tip growth velocities as a function of undercooling is required. A milestone in solidification research was the Isothermal Dendritic Growth Experiment (IDGE) performed on transparent alloy systems in microgravity conditions during a space shuttle flight in 1994 [11]. By eliminating buoyancyinduced convection, reliable testing of the diffusion-limited growth theory was possible for the first time [12-16]. Direct measurements of tip velocities and tip radii of succinonitrile dendrites as a function of undercooling were used to validate the previously assumed general applicability of Ivantsov's transport solution that relates the total undercooling to the product of tip velocity and tip radius (Péclet number) [17].

*maike.becker@dlr.de

${ }^{\dagger}$ Present address: Deutsche Gesellschaft für Materialkunde DGM e.V., 10179 Berlin, Germany.

†florian.kargl@dlr.de
Although transparent organics such as succinonitrile $\left[\mathrm{NC}\left(\mathrm{CH}_{2}\right)_{2} \mathrm{CN}\right]$ and pivalic acid $\left[\left(\mathrm{CH}_{3}\right)_{3} \mathrm{CCO}_{2} \mathrm{H}\right]$ are good metal analogs to study dendritic growth in terms of pattern formation and evolution, some of their physicochemical properties differ distinctively from those found in metals. Especially, thermal properties such as conductivity, diffusivity, and freezing temperatures are not comparable with metal systems as they are one or several orders of magnitude lower $[12,18,19]$. Hence, as physicochemical properties have a strong influence on the growth process, directly transferring results of the IDGE experiments to solidifying metal alloys is difficult.

Therefore, benchmark experiments on metals are needed that provide tip growth velocities over a sufficiently large range of undercooling. For deeply undercooled melts $(\Delta T=$ 20-325 K) tip velocity measurements are available for many different metallic alloys [20-23]. To achieve high undercoolings, typically levitation techniques or the glass fluxing method are used [24,25]. The temperature of the melt is monitored by pyrometers and growth velocities are measured by tracking the recalescence front that develops due to released latent heat resulting from solidification [26]. For near-equilibrium solidification and low undercoolings the contrast between the melt and the recalescence front is not strong enough to be monitored. Furthermore, the developing microstructure cannot be observed in situ.

To reveal dynamic solidification phenomena in metallic alloys processed under near-equilibrium conditions, x-ray diagnostics is the method of choice [27]. X-ray radiography provides a two-dimensional (2D) representation of the $\mathrm{x}$ rayed objects wherefore usually thin samples $(\leqslant 200 \mu \mathrm{m})$ are investigated [27-30]. Synchrotron x-ray sources yield excellent results [31-35], but the orientation of the $x$-ray beam and thus the orientation of the sample is predefined. Bogno et al. [32] used synchrotron radiation to investigate growth rates of dendrites in an $\mathrm{Al}-10 \mathrm{wt}$ \% $\mathrm{Cu}$ alloy. The samples were processed in a Bridgman-type furnace operating in the isothermal mode. With decreasing sample temperature they measured an increase of tip growth velocities for the first 
part of dendritic growth. They interpreted this accelerating growth regime to represent free growth. A comparison of the accelerating growth regime with analytical relationships for free dendritic growth [36,37] yielded good agreement. However, the comparability with the model reduces to a few data points only due to the rather short accelerating tip growth velocity regime measured (between 5 and $14 \mu \mathrm{m} \mathrm{s}^{-1}$ ) and, moreover, the impact of the vertical sample orientation on the redistribution of the solute around the tips remains unclear. In binary alloys the tip growth is mainly governed by the distribution of the solutal diffusion field around the tip. Sedimentation of the dense solute as a result of a vertical sample orientation can considerably influence the tip kinetics [38].

Laboratory microfocus x-ray sources have the advantage of orienting the thin sample plane perpendicular to the gravitational force. In a benchmark microgravity study on equiaxed isothermal solidification in $\mathrm{Al}-\mathrm{Cu}$, no significant differences in terms of nucleation density and growth characteristics with respect to an on-ground horizontal reference experiment were detected [39]. Thus, gravity-induced fluid flow is minimized and the diffusion field is comparable to microgravity conditions, at least for the isothermal case. This is an important aspect for the comparability of the experiments to diffusionlimited growth models [40].

We conduct solidification experiments in Al-Ge alloys in a horizontal sample alignment minimizing gravity-induced fluid flow to analyze the tip kinetics. The repetition of the experiments selecting one Al-Ge alloy composition enables us to obtain free dendritic tip velocities over one order of magnitude. Thus, a large data set of dendritic tip growth velocities is generated, which is needed for a stringent test of growth models. To basically evaluate the measurements, we compare our data to the model predictions of the isothermal version of the Lipton-Glicksman-Kurz (LGK) model [41]. The LGK model was developed to predict the tip growth rates of equiaxed dendrites in low undercooled melts and is based on Ivantsov's transport solution. The driving force for solidification is the total undercooling at the tip that can be expressed as the sum of thermal, solutal, and curvature undercooling, $\Delta T=\Delta T_{T}+\Delta T_{C}+\Delta T_{R}$. In principle, the model predicts faster growth rates for increasing melt undercoolings, wherefore the absolute values strongly depend on the respective material parameters. The fundamental derivations of this model are often used to predict the tip kinetics in more complex models of equiaxed solidification or to evaluate experimental measurements or numerical simulations [36,37,42-47]. We discuss the agreement of the free dendritic tip growth velocity data with the LGK model and the implication on a possible influence of the sample thickness.

The solidification experiments are performed in a nearisothermal furnace [48] with a constant cooling rate of $1 \mathrm{~K}$ $\min ^{-1}$. The furnace cartridge is implemented in the $\mathrm{x}$-ray radiography facility called X-RISE [49] designed around a microfocus transmission $\mathrm{X}$-ray source from FineTec FineFocus Technologies GmbH. For x-ray generation a single-crystal diamond transmission target coated with tungsten is used. To achieve good image quality and contrast, we use an operating voltage and power of $70 \mathrm{kV}$ and $134 \mu \mathrm{A}$, respectively. The detector is a Vosskuehler 11000 with a $24 \mathrm{~mm} \times 36 \mathrm{~mm}$
CCD sensor and a structured CsI scintillator. The CCD sensor records $4024 \times 2860$ pixel, 12-bit images with a native pixel size of $9 \mu \mathrm{m}$. A sample-to-detector magnification of $\approx 2.3$ leads to a virtual pixel size of $3.9 \mu \mathrm{m}$. The field of view has a diameter of $10.5 \mathrm{~mm}$.

Dendrite tip growth rates are measured in five samples of the alloy composition Al-24 at. \% Ge. The samples are cast from pure aluminum $(5 N)$ and semiconductor-grade germanium. The cast ingots with a diameter of $12 \mathrm{~mm}$ are sectioned in disks and are ground to achieve the desired thickness. Samples No. 1 and No. 2 exhibit a thickness of $200 \mu \mathrm{m}$, whereas samples No. 3-No. 5 exhibit a thickness of $350 \mu \mathrm{m}$. Due to the different $\mathrm{x}$-ray absorptions of aluminum and germanium, solid and liquid phases can be distinguished in the radiography images, and the microstructure evolution on solidification is continuously monitored. In between four and eight cooling cycles are performed for each sample. The number of dendrites nucleating in each sample varies between one and 18. As the grain density is quite low, some dendrites can develop severalmillimeter-long arms which bring major benefits when it comes to growth rate measurements. A large distance between the nucleating dendrites is also favorable in order to minimize the mutual thermal and solutal influence of the dendrites. With the resolution of the $\mathrm{x}$-ray facility, the growth rates of the tips can be tracked reliably when the arm length exceeds $200 \mu \mathrm{m}$. The dendrite tips are tracked with the IMAGEJ plugin "Manual Tracking" [50]. The velocities are then calculated using the arm length measurements and assuming a constant velocity between three consecutive images wherefore one image corresponds to $5 \mathrm{~s}$ of experiment time.

In Fig. 1(a) a radiography image of sample No. 1, cooling cycle No. 1, is depicted. Ten dendrites nucleate during this cooling cycle. The tip velocities of the three fastest growing dendrite tips are plotted against time in Fig. 1(b). Time $t_{0}=0$ is the point in time when the first dendrite nucleates in the sample. This includes all cooling cycles. All three selected dendrite tips show a velocity increase with time in the beginning, a maximum in velocity, followed by a decrease. The differences in growth rate maxima reflect variable growth conditions. A detailed look at the individual tips shows that the velocities are dependent on the respective dendrite tip surrounding. When we compare the tip velocities of the dendrite arms D1-1 and D1-2, it becomes clear that the early impingement of D1-2 with the neighboring dendrite leads to an earlier growth rate decrease compared to dendrite arm D1-1. Consequently, dendrite arm D1-1 shows a prolonged acceleration of tip velocity. Dendrite D2 nucleates about $159 \mathrm{~s}$ later than dendrite D1. As the melt has cooled down by $2.7 \mathrm{~K}$ at this instance, the tip velocity of dendrite arm D2-1 is considerably faster compared to the arms of dendrite D1 after nucleation because the driving force for solidification is higher.

Considering all growth rate measurements, two different growth behaviors can be distinguished: (a) accelerating tip growth followed by (b) decelerating tip growth. The accelerating tip growth regime corresponds to free dendritic growth which is driven by a constant increase in undercooling resulting from sample cooling. The decelerating tip growth regime is probably due to soft impingement of the solutal fields from neighboring dendrites that diffuse into the liquid. An increase of the solute concentration in front of a dendrite tip lowers 

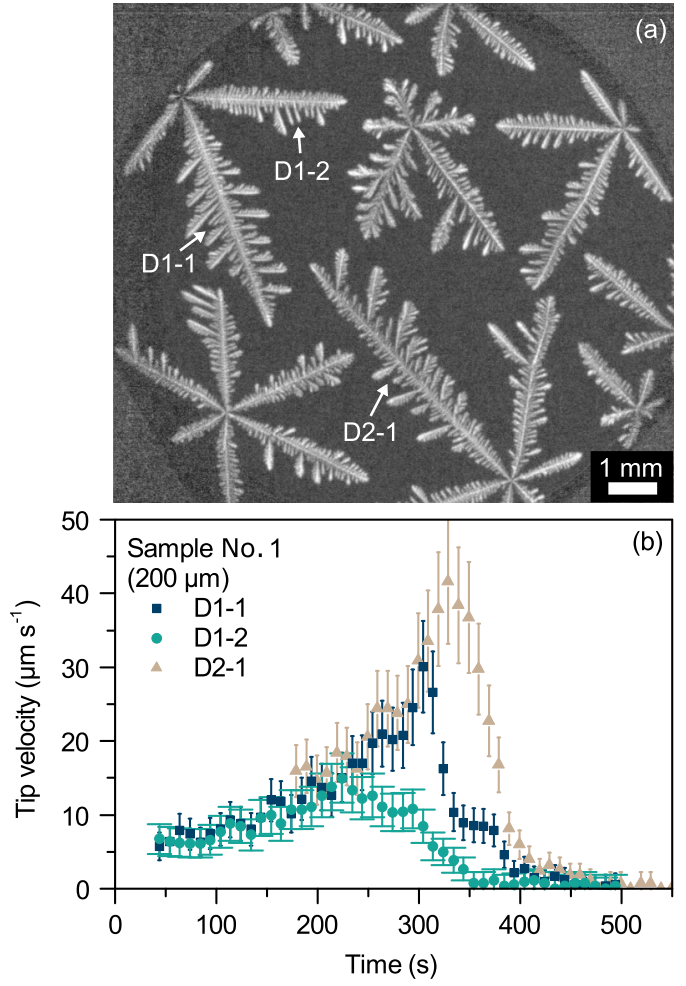

FIG. 1. (a) X-ray radiography image of Al-24 at. \% Ge sample No. 1 , cooling cycle No. 1 , recorded $654 \mathrm{~s}$ after nucleation. (b) Tip growth rates of the three fastest growing dendrite tips plotted against time. A velocity acceleration phase is observed for all three dendrite tips. The error bars are calculated by propagating the error of the length measurement $( \pm 30 \mu \mathrm{m})$ and additionally using an uncertainty of $2 \mathrm{~s}$ for the time measurement. A temporal uncertainty is included to account for the larger measurement inaccuracies resulting from increasing growth velocities.

the constitutional undercooling $\Delta T_{C}$ and hence the tip growth velocity. Synchrotron studies on $\mathrm{Al}-\mathrm{Cu}$ and $\mathrm{Al}-\mathrm{Ge}$ alloys have shown that the deceleration of tip velocities is correlated with an increase of the solutal far-field concentration [32,34].

In fact, we observe many dendrite tips that show no prolonged accelerating growth regime. This indicates that a solutal field interaction is already present at the early growth stages. A similar observation was made in a horizontal equiaxed solidification experiment by Murphy et al. [42] in an Al-20 wt. $\% \mathrm{Cu}$ alloy. The dendrite arms with a maximum length of $1.1 \mathrm{~mm}$ rarely showed an accelerating growth regime. As an unambiguous identification of the accelerating phase is necessary for the interpretation of our data, as discussed hereafter, we decided to use only dendrite tips for the analysis that show clear accelerating trends. In consequence, we selected the fastest growing dendrite tips exhibiting total arm lengths of more than $3 \mathrm{~mm}$.

In Fig. 2(a) solely the accelerating growth velocities of selected dendrite tips of all five samples are plotted against time. Time $t_{0}=0$ corresponds to the first dendrite appearance in the respective sample. Tip velocity maxima of all experiments vary between 30 and $140 \mu \mathrm{m} \mathrm{s}^{-1}$. For all five individual samples a clear trend of increasing tip velocities with time is detected. The more time passes, the higher are the measured tip
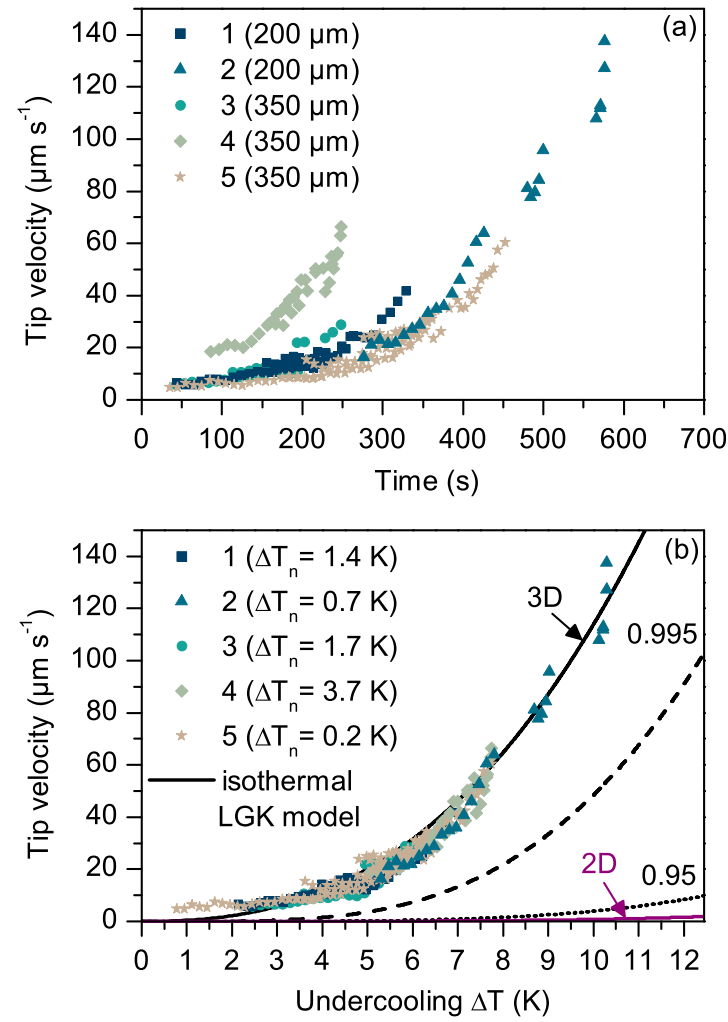

FIG. 2. (a) Accelerating tip growth rates measured in five different samples of the alloy composition Al-24 at. \% Ge. Time $t_{0}=0$ corresponds to the first dendrite appearance in the respective sample. (b) Tip growth velocities against undercooling. An addition of different amounts of initial nucleation undercooling for every sample leads to a single master curve which is in line with growth theory. The curves are modeled with the isothermal version of the LGK model using the model parameters listed in Table I. The solid lines represent the three-dimensional and two-dimensional case as indicated. The dotted lines represent the solution for an elliptical dendrite tip using aspect ratios $A(0<A<1)$ of 0.95 and 0.995 .

velocities. Although all growth rates show the same increasing trend, tip velocities are different for the same point in time after $t_{0}$. There is no physical correlation between tip velocity and time. However, a correlation between tip velocities and undercooling exists.

Undercooling is the temperature difference between the actual temperature $T$ of the liquid and the liquidus temperature $T_{L}$ of the initial composition $\Delta T=\left(T_{L}-T\right)$. The constant cooling rate applied in the experiments corresponds to a constant increase in melt undercooling. Therefore, the experiment time can be directly converted into undercooling. Owing to the near-isothermal sample environment, the driving force for solidification is the uniform melt undercooling that increases with the applied cooling rate by $1 \mathrm{~K} \mathrm{~min}^{-1}$. From the in situ experiments it can be seen that the dendrites nucleate at various times and consequently at different undercoolings. The highest nucleation undercooling for a dendrite is found in sample No. 2. Two dendrites in cooling cycle No. 6 nucleate in a melt that is undercooled by at least $9.9 \mathrm{~K}$. Consequently, these dendrites show the fastest tip velocities of all investigated tips [cf. Fig. 2(a)]. 
As it is unlikely that the nucleation temperature $T_{0}$ of the first dendrite in all samples directly corresponds to $T_{L}$, an initial nucleation undercooling $\Delta T_{n}$ must exist in all of the experiments. The amount of $\Delta T_{n}$ for the samples can be estimated by using knowledge from growth theory postulating that in a particular alloy a dendrite tip grows with a certain velocity at a certain undercooling. In this context, it is obligatory that the accelerating tip growth regimes of all investigated samples follow a single master curve. As the individual curves show the same increasing slope, a convergence of all experiment curves to a single master curve can be achieved by adding between 0.2 and $3.7 \mathrm{~K}$ of $\Delta T_{n}$ [cf. Fig. 2(b)].

The measured data showing a large variation of tip velocities allow for the adjustment of the curves so that the nucleation undercooling for each individual sample can be derived assuming that at least one of the samples solidifies with only a minor nucleation undercooling. As it is probable that even for the earliest nucleating dendrite among all of the measurement an additional small nucleation undercooling exists, a small value of $0.2 \mathrm{~K}$ was chosen for sample No. 5. Unfortunately, an absolute determination of the sample temperature is challenging as the thermocouple is positioned $3.6 \mathrm{~mm}$ away from the sample to avoid disturbances of the isothermal field of the furnace. With a calibration procedure the temperature can be estimated, but only with an accuracy that is insufficiently accurate for the analysis. The latter is a general and so far unresolved problem for these types of experiments. In consequence, this means an uncertainty regarding the point of zero undercooling remains. However, the procedure to process a number of samples to assess the relative undercooling is in our understanding to date the best approach to calibrate the absolute value of undercooling.

Due to the horizontal sample alignment, gravity-driven fluid flow is minimized inside the samples and tip growth under neardiffusive conditions is achieved. This allows us to compare the data to a diffusion-limited growth model. However, as local convection in front of the tips is still possible, an impact on the tip growth rates cannot be ruled out completely. For this to be finally answered, benchmark experiments would have to be carried out in microgravity conditions.

The tip radii of the dendrites are a lot smaller than the sample thickness, at least for intermediate and fast growth rates. Hence, in a first instance, their growth is assumed to represent three-dimensional (3D) conditions. However, the small sample thickness confines the diffusion of the solute around the dendrite tips in the $z$ direction. It is known that $2 \mathrm{D}$ tip growth is considerably slower than $3 \mathrm{D}$ tip growth $[47,53]$. The reason lies in the confined distribution of the diffusion field around the tip. In 2D the rejected solute cannot distribute in the $z$ direction, thus the solute enriches disproportionately in the $x$ and $y$ directions. This leads to slower tip growth rates in $2 \mathrm{D}$ compared to $3 \mathrm{D}$.

To evaluate a possible effect of the sample thickness on dendrite tip growth rates, we measured the tip velocities in 200and in 350- $\mu$ m-thick samples. We found comparable growth velocities of the dendrite tips for a certain undercooling, as indicated by all data collapsing on a single master curve. This is an argument for the minor influence of the sample confinement on the tip velocities in the present experiments. This argument is supported by the study of Bogno et al. [32], who measured dendritic tip velocities in 200- $\mu$ m-thin $\mathrm{Al}-10$ wt. \% Cu alloys, finding good agreement with a 3D analytical relationship for free dendritic growth [36,37].

We compare the velocity versus undercooling data to the isothermal version of the LGK model. Thermal undercooling $\Delta T_{T}$ is neglected as fast thermal diffusion is assumed. Then a free tip grows at an undercooling of

$$
\Delta T=T_{L}\left(C_{0}\right)-T^{*}=\Delta T_{C}+\Delta T_{R} .
$$

The dendrite tip is considered to be a paraboloid of revolution with temperature $T^{*}$ in the liquid at the interface. In a binary alloy the composition difference $\Delta C=C_{L}^{*}-C_{0}$ at the interface can be expressed in temperatures according to the phase diagram and is called the constitutional undercooling,

$$
\Delta T_{C}=m\left(C_{0}-C_{L}^{*}\right),
$$

where $m$ is the liquidus slope, $C_{0}$ is the initial alloy composition, and $C_{L}^{*}$ is the solute composition at the solid-liquid interface.

The solute field around the tip is given by the Ivantsov solution,

$$
\operatorname{Iv}\left(P_{C}\right)=P_{C} \exp \left(P_{C}\right) E_{1}\left(P_{C}\right)=\frac{C_{L}^{*}-C_{0}}{C_{L}^{*}(1-k)} .
$$

$P_{C}=v R / 2 D$ is the solutal Péclet number, $D$ is the solute diffusivity, $E_{1}$ is the exponential integral function, and $k$ is the partition coefficient. Rearranging Eq. (3) gives for the composition at the interface, $C_{L}^{*}=C_{0} /\left[1-(1-k) \operatorname{Iv}\left(P_{C}\right)\right]$.

Using the Gibbs-Thomson relation for the curvature undercooling $\Delta T_{R}$, Eq. (1) can be written as

$$
\Delta T=m C_{0}\left(1-\frac{1}{1-(1-k) \operatorname{Iv}\left(P_{C}\right)}\right)+\frac{2 \Gamma}{R} .
$$

As there is no unique solution for the radius $R$ and the velocity $v$ of a dendrite tip, the LGK model uses a selection criterion of

$$
R=\frac{\Gamma}{\sigma^{*}}\left(\frac{1}{2 m P_{C} C_{L}^{*}(1-k)}\right) .
$$

The selection constant $\sigma^{*}$ defines the operating state of a dendrite tip and is dependent on the anisotropy of the solidliquid interfacial energy according to microscopic solvability theory [54]. As no measurements of the anisotropy parameters in the Al-Ge system are available, the $\sigma^{*}$ value is used as a free parameter. With the thermophysical parameters of Table I and a selection constant of $\sigma^{*}=0.024$, Eqs. (4) and (5) can be solved and the solid black model curve in Fig. 2(b) is derived. Interestingly, the experimental data are best reproduced by using a selection constant that is close to the value of standard marginal stability $\left(\sigma^{*}=1 / 4 \pi^{2}[55]\right)$. Especially, if the high velocity regime of sample No. 2 is taken into account, the agreement between the experiments and the model is significant. Nevertheless, some deviations from the model predictions are found in the low undercooling regime $\Delta T<2 \mathrm{~K}$ and in the intermediate undercooling regime $2 \mathrm{~K}<\Delta T<8 \mathrm{~K}$. The discrepancies might be attributable to model assumptions that are not met in experiment reality. At the beginning of growth, thermal diffusion could play a pronounced role. As the dendrites nucleate in an already by a few degrees undercooled melt, fast thermal heat release could promote growth rates that 
TABLE I. Thermophysical parameters for the alloy composition Al-24 at. \% Ge. The liquidus temperature, the solute partition coefficient, and the liquidus slope are derived from the equilibrium phase diagram [51].

\begin{tabular}{lc}
\hline \hline Property & Value \\
\hline Initial composition, $C_{0}$ & Al-24 at. $\% \mathrm{Ge}$ \\
Liquidus temperature, $T_{L}\left(C_{0}\right)$ & $732 \mathrm{~K}$ \\
Liquidus slope, $m$ & $-10.4 \mathrm{~K}$ at. $\%{ }^{-1}$ \\
Solute partition coefficient, $k$ & 0.11 \\
Solute diffusion coefficient, $D$ & $7\left(10^{-9} \mathrm{~m}^{2} \mathrm{~s}^{-1}\right)^{\mathrm{a}}$ \\
Gibbs-Thomson coefficient, $\Gamma$ & $1.52\left(10^{-7} \mathrm{~K} \mathrm{~m}\right)^{\mathrm{b}}$ \\
\hline \hline
\end{tabular}

$\overline{{ }^{a} \text { Estimated value based on an in situ } \mathrm{x} \text {-ray radiography linear shear- }}$ cell interdiffusion experiment at DLR.

${ }^{\mathrm{b}}$ Reference [52].

are higher compared to steady-state growth conditions. On the other hand, the confinement of the dendrites could lead to slower growth rates than predicted by the $3 \mathrm{D}$ steady-state growth model. The combination of the thickness of the sample and of the thickness of the solute boundary layer $D / v$ plays a significant role. If the solute boundary layer is larger than the sample thickness, the diffusion field gets perturbed, which leads to reduced growth velocities. The diffusion length for the experiments presented here is between 50 and $350 \mu \mathrm{m}$. This effect could be attributable to the differences between the experimental measurements and the model predictions in the intermediate undercooling regime.

Moreover, the shape of the tip can considerably influence the growth velocities. It was already shown in a phase-field approach that a small sample thickness can modify the tip shape and the diffusion field [56]. Due to the thin sample confinement, the tips can become less axisymmetric. To access the influence of the sample thickness, the isothermal LGK model for the 2D case is additionally shown in Fig. 2(b). We model the solution for a twofold elliptical paraboloid following the analysis of McFadden and Browne [57] and Horvay and Cahn [58]. They use a modified Ivantsov function that includes a shape factor $B$,

$$
\operatorname{Iv}\left(P_{C}, B\right)=P_{C} \exp \left(P_{C}\right) \sqrt{1+\frac{B}{P_{C}}} J\left(P_{C}, B\right) .
$$

The function $J(x, B)$ is an integral function given by

$$
J\left(P_{C}, B\right)=\int_{P_{C}}^{\infty} \frac{\exp (-x)}{\sqrt{x(B+x)}} d x,
$$

with $A=1 / \sqrt{1+B}$. The solution for a $2 \mathrm{D}$ dendrite plate is given when $A=0$ and the solution for a 3D paraboloid of revolution is given when $A=1$. Additionally, the $2 \mathrm{D}$ formulation for $\Delta T_{R}=\Gamma / R$ is used.

The tip velocity for the $2 \mathrm{D}$ case is much slower than for the 3D case. Even if we assume that we underestimated the initial nucleation undercooling, it is clear that the tip velocities are far away from the $2 \mathrm{D}$ case. In Fig. 2(b) the solutions for $A=0.995$ and $A=0.950$ are plotted. Even by considering the tip velocity curves of an elliptical dendrite tip, the experimentally measured data agree best with the $3 \mathrm{D}$ case. Hence, a strong influence of the confinement was not found in this case. However, the impact of the sample thickness on solidification patterns must always be considered and carefully investigated.

The tip velocity determination over a wide range of global undercooling $(1-10 \mathrm{~K})$ makes the measurements presented here a unique data set for metallic alloys. The key elements of the measurements and the analysis are as follows: (a) to repeatedly execute the solidification experiment with one sample to get one tip velocity-against-undercooling curve, and (b) to repeat the same experiment with several samples of the same alloy composition to achieve an overlap of the individual tip velocity curves by evaluating the initial nucleation undercooling for each sample. The superposition of all curves to a single master curve is possible because the measured tip growth velocities cover one order of magnitude. As the data are adequately reproduced by the $3 \mathrm{D}$ isothermal LGK model, it can be used for the verification of numerical growth models that take into account the active cooling of a sample. Furthermore, the measurement approach presented here can be applied to other alloy systems (Al-Cu, Al-Ni) often used as model systems to derive growth rates and other grain characteristics. However, two major challenges remain: (a) to better determine the initial nucleation undercooling, and (b) to better determine the influence of the remaining fluid flow inside the sample, for example, in a microgravity experiment.

We thank Dr. Laszlo Sturz from Access e.V. in Aachen, Germany, for his calculation of the Al-Ge equilibrium phase diagram using CALPHAD. We thank Hydro Aluminium Deutschland in Bonn, Germany, and the Leibnitz-Institut für Kristallzüchtung (IKZ) in Berlin, Germany, for kindly providing aluminum and germanium, respectively. Sincere thanks are given to our colleagues from DLR, Dr. Elke Sondermann for determining the solute diffusion coefficient and Prof. Dr. Andreas Meyer, Dr. Sonja Steinbach, and Dr. Matthias Kolbe for critically reading this manuscript.
[1] W. Kurz and D. J. Fisher, Fundamentals of Solidification, 3rd ed. (Trans Tech, Stafa-Zurich, Switzerland, 1992).

[2] B. Chalmers, Principles of Solidification (Wiley, New York, 1964).

[3] R. Trivedi and W. Kurz, Int. Mater. Rev. 39, 49 (1994).

[4] M. Rappaz, Int. Mater. Rev. 34, 93 (1989).

[5] D. Tourret and A. Karma, Acta Mater. 120, 240 (2016).
[6] M. Založnik, A. Viardin, Y. Souhar, H. Combeau, and M. Apel, IOP Conf. Ser.: Mater. Sci. Eng. 84, 012074 (2015).

[7] Y. Souhar, V. F. De Felice, C. Beckermann, H. Combeau, and M. Založnik, Comput. Mater. Sci. 112, 304 (2016).

[8] A. Karma and D. Tourret, Curr. Opin. Solid State Mater. Sci. 20, 25 (2016). 
[9] J. Lipton, M. E. Glicksman, and W. Kurz, Mater. Sci. Eng. 65, 57 (1984).

[10] P. Pelcé, Dynamics of Curve Fronts (Academic, New York, 1988).

[11] M. E. Glicksman, R. C. Hahn, T. A. Lograsso, S. H. Tirmizi, and E. Winsa, Metall. Trans. A 19, 1945 (1988).

[12] M. B. Koss, J. C. LaCombe, L. A. Tennenhouse, M. E. Glicksman, and E. A. Winsa, Metall. Mater. Trans. A 30, 3177 (1999).

[13] M. E. Glicksman, M. B. Koss, L. T. Bushnell, J. C. LaCombe, and E. A. Winsa, ISIJ Int. 35, 604 (1995).

[14] M. E. Glicksman, M. B. Koss, and E. A. Winsa, Phys. Rev. Lett. 73, 573 (1994).

[15] J. C. LaCombe, M. B. Koss, and M. E. Glicksman, Metall. Mater. Trans. A 38, 116 (2007).

[16] M. E. Glicksman and A. O. Lupulescu, J. Cryst. Growth 264, 541 (2004).

[17] G. P. Ivantsov, Dokl. Akad. Nauk SSSR 58, 567 (1947).

[18] M. A. Chopra, M. E. Glicksman, and N. B. Singh, J. Cryst. Growth 92, 543 (1988).

[19] A. Ludwig, Acta Mater. 44, 3643 (1996).

[20] R. Kobold, W. W. Kuang, H. Wang, W. Hornfeck, M. Kolbe, and D. M. Herlach, Philos. Mag. Lett. 97, 249 (2017).

[21] Y. Ruan, S.-Y. Chang, and M. Dao, Cryst. Growth Des. 15, 5661 (2015).

[22] D. M. Herlach, Crystals 5, 355 (2015).

[23] H. Wang, D. M. Herlach, and R. P. Liu, Europhys. Lett. 105, 36001 (2014).

[24] D. M. Herlach, Metals 4, 196 (2014).

[25] Y. Ruan and F. P. Dai, Intermetallics 25, 80 (2012).

[26] Q. Wang, L.-M. Wang, M. Z. Ma, S. Binder, T. Volkmann, D. M. Herlach, J. S. Wang, Q. G. Xue, Y. J. Tian, and R. P. Liu, Phys. Rev. B 83, 014202 (2011).

[27] R. H. Mathiesen, L. Arnberg, H. Nguyen-Thi, and B. Billia, JOM 64, 76 (2012).

[28] A. J. Clarke, D. Tourret, S. D. Imhoff, P. J. Gibbs, K. Fezzaa, J. C. Cooley, W. K. Lee, A. Deriy, B. M. Patterson, P. A. Papin, K. D. Clarke, R. D. Field, and J. L. Smith, Adv. Eng. Mater. 17, 454 (2015).

[29] H. Yasuda, T. Nagira, M. Yoshiya, N. Nakatsuka, A. Sugiyama, K. Uesugi, and K. Umetani, ISIJ Int. 51, 402 (2011).

[30] E. W. J. Miller and J. Beech, Metallography 5, 298 (1972).

[31] W. U. Mirihanage, K. V. Falch, I. Snigireva, A. Snigirev, Y. J. Li, L. Arnberg, and R. H. Mathiesen, Acta Mater. 81, 241 (2014).

[32] A. Bogno, H. Nguyen-Thi, G. Reinhart, B. Billia, and J. Baruchel, Acta Mater. 61, 1303 (2013).

[33] G. Reinhart, C.-A. Gandin, N. Mangelinck-Noël, H. NguyenThi, J.-E. Spinelli, J. Baruchel, and B. Billia, Acta Mater. 61, 4765 (2013).
[34] S. McFadden, P. L. Schaffer, R. H. Mathiesen, and D. J. Browne, Mater. Sci. Forum 654-656, 1359 (2010).

[35] H. Nguyen-Thi, G. Reinhart, N. Mangelinck-Noël, H. Jung, B. Billia, T. Schenk, J. Gastaldi, J. Härtwig, and J. Baruchel, Metall. Mater. Trans. A 38, 1458 (2007).

[36] C. Y. Wang and C. Beckermann, Metall. Mater. Trans. A 25, 1081 (1994).

[37] P. Pelcé and Y. Pomeau, Stud. Appl. Math. 74, 245 (1986).

[38] N. Shevchenko, O. Roshchupkina, O. Sokolova, and S. Eckert, J. Cryst. Growth 417, 1 (2015).

[39] A. G. Murphy, R. H. Mathiesen, Y. Houltz, J. Li, C. Lockowandt, K. Henriksson, N. Melville, and D. J. Browne, J. Cryst. Growth 454, 96 (2016).

[40] S. Akamatsu and H. Nguyen-Thi, Acta Mater. 108, 325 (2016).

[41] J. Lipton, M. E. Glicksman, and W. Kurz, Metall. Trans. A 18, 341 (1987).

[42] A. G. Murphy, R. H. Mathiesen, Y. Houltz, J. Li, C. Lockowandt, K. Henriksson, G. Zimmermann, N. Melville, and D. J. Browne, J. Cryst. Growth 440, 38 (2016).

[43] Q. Li and C. Beckermann, J. Cryst. Growth 236, 482 (2002).

[44] M. A. Chopra, M. E. Glicksman, and N. B. Singh, Metall. Mater. Trans. A 19, 3087 (1988).

[45] J. C. Ramirez and C. Beckermann, Acta Mater. 53, 1721 (2005).

[46] A. J. Melendez and C. Beckermann, J. Cryst. Growth 340, 175 (2012).

[47] M. Eshraghi, M. Hashemi, B. Jelinek, and S. D. Felicelli, Metals 7, 474 (2017).

[48] M. Becker, C. Dreißigacker, S. Klein, and F. Kargl, Rev. Sci. Instrum. 86, 063904 (2015).

[49] S. Klein, D. Bräuer, M. Becker, A. Knipstein, S. Meckel, E. Sondermann, and F. Kargl, Int. J. Microgravity Sci. Appl. 33, 330405 (2016).

[50] F. Cordelières, https://imagej.nih.gov/ij/plugins/manual (2004).

[51] F. Islam, A. K. Thykadavil, and M. Medray, J. Alloys Compd. 425, 129 (2006).

[52] N. Yan, F.-P. Dai, W.-L. Wang, and B.-B. Wei, Chin. Phys. Lett. 28, 078101 (2011).

[53] L. Yuan and P. D. Lee, Modell. Simul. Mater. Sci. Eng. 18, 055008 (2010).

[54] D. A. Kessler, J. Koplic, and H. Levine, Adv. Phys. 37, 255 (1988).

[55] J. S. Langer and H. Müller-Krumbhaar, Acta Metall. 26, 1681 (1978).

[56] A. J. Clarke, D. Tourret, Y. Song, S. D. Imhoff, P. J. Gibbs, J. W. Gibbs, and K. Fezzaa, Acta Mater. 129, 203 (2017).

[57] S. McFadden and D. J. Browne, Comput. Mater. Sci. 55, 245 (2012).

[58] G. Horvay and J. W. Cahn, Acta Metall. 9, 695 (1961). 